Proceedings of the XXXVIII International School and Conference on the Physics of Semiconductors "Jaszowiec" 2009

\title{
Phonon-Induced Pure Dephasing of Two-Electron Spin States in Vertical Quantum Dot Molecules \\ K. RosZAK ${ }^{a, b, *}$ AND P. MACHNIKOWSKI ${ }^{b}$
}

${ }^{a}$ Department of Condensed Matter Physics, Faculty of Mathematics and Physics, Charles University 12116 Prague, Czech Republic

${ }^{b}$ Institute of Physics, Wrocław University of Technology, Wybrzeże Wyspiańskiego 27, 50-370 Wrocław, Poland

Phonon-induced dephasing of two-electron spin states in two vertically stacked self-assembled GaAs/InGaAs quantum dots is studied. A pure dephasing process due to elastic phonon scattering is found to dominate at low temperatures. This process is independent of the spin-orbit coupling and does not require the presence of a magnetic field. It relies on interdot tunneling and the Pauli principle, which make the double quantum dot gate for spin based quantum computing possible, and therefore cannot be avoided.

PACS numbers: 73.21.La, 03.65.Yz, 72.10.Di, 03.67.Lx

\section{Introduction}

Phonon-induced decoherence has turned out to be an unavoidable problem in the realization of quantum dot (QD) based quantum computing. The carrier-phonon interaction leads to pure dephasing of charge-state superpositions on picosecond timescales. One of the possible solutions to this problem is to use the electron spin degrees of freedom for information storage [1] or as the qubit itself [2-4]. Phonon influence on spin states is much weaker than on charge states due to the lack of a direct spin-phonon coupling (an interaction is mediated by the spin-orbit coupling which is weak in QDs and strongly dependent on the magnitude of the magnetic field). Experimentally reported relaxation times of a single spin in a QD are of the order of milliseconds $[5,6]$ and are promising compared to the coherent control rates achieved.

In this contribution, we study phonon-induced decoherence of singlet-triplet superpositions in two vertically coupled self-assembled quantum dots. In a recent work [7], we have shown that the carrier-phonon interaction which, apparently, affects only charge states, in fact destroys also two-electron spin coherence in tunnel-coupled QDs. This dephasing results from elastic phonon scattering and spin-dependent Pauli blocking of certain virtual transitions. Here we apply our model to a self-assembled InAs/GaAs double dot. We show that due to large Coulomb energies in a strongly confined self-assembled structure, the elastic scattering process is by far the major contribution to spin dephasing in this system.

The physical mechanism responsible for this dephasing channel is as follows. In the lowest energy state of the two-electron system, each QD is occupied by a single electron. The spin configuration of the system may then be either singlet or triplet. In the former case, the orbital wave function is symmetric and a transition to a higher-energy doubly occupied state is possible. In the triplet configuration this is forbidden by the Pauli principle. Although such transitions are inefficient at low temperatures, two-phonon elastic scattering is still possible, in which absorption of a phonon is followed by re-emission of another one $[8,9]$. In such a process, the doubly occupied state is involved only "virtually", so the phonons can have arbitrary (but equal) energies. Therefore, even at low temperatures, phonons scatter on a double QD in the singlet state, while the triplet state is transparent to them. This distinguishability leads to pure dephasing of any singlet-triplet superposition, in some sense analogous to the "collisional decoherence" of the orbital degrees of freedom [10, 11].

\section{Model}

In the calculations, we take into account the three lowest-energy singlet states (the one where the QDs are each occupied by one electron in the ground state and the two doubly occupied states) and one of the triplet states. The explicit forms of these states before and after the diagonalization of the double quantum dot (DQD) Hamiltonian, as well as the Hamiltonian itself may be found in Ref. [7]. The carrier-phonon interaction is described by

$$
H_{\mathrm{int}}=\sum_{s, i} \sum_{\boldsymbol{k}} F_{i}(\boldsymbol{k}) a_{i s}^{\dagger} a_{i s}\left(b_{\boldsymbol{k}}+b_{-\boldsymbol{k}}^{\dagger}\right) \text {, }
$$

where $s=\uparrow, \downarrow$ is the spin index and $i=u, d$ denotes the upper and lower QD. $a_{i s}^{\dagger}, a_{i s}$ are creation and annihilation operators for an electron with spin $s$ located in dot $i$, while $b_{\boldsymbol{k}}$ and $b_{\boldsymbol{k}}^{\dagger}$ are phonon creation and annihilation operators. $F_{u / d}(\boldsymbol{k})=F(\boldsymbol{k}) \exp \left( \pm \mathrm{i} k_{z} D / 2\right)$ are coupling constants for the upper and lower dots, respectively, and $D$ is the interdot distance. We include the deformation potential and piezoelectric coupling following Ref. [1, 12]. 


\section{Results}

We investigate phonon-induced dephasing of a superposition of the spin triplet state and the singlet state which is lowest in energy. The information about the phase of this superposition is stored in the appropriate off-diagonal element of the reduced density matrix of the two-electron system $\rho_{\mathrm{DQD}}$. Its evolution was calculated using the time-convolutionless (TCL) projection operator method in the Markov limit [13] and the resulting dephasing rates are plotted in Fig. 1. Parameters typical for vertically stacked, self-assembled GaAs/InGaAs QDs were used. The dots were assumed to be identical with the in-plane width $l_{\perp}=5 \mathrm{~nm}$ and the width in the growth direction $l_{z}=2 \mathrm{~nm}$, the interdot distance was taken to be $D=12 \mathrm{~nm}$. The energy difference between singly and doubly occupied QDs was fixed at $V=5 \mathrm{meV}$. Material parameters used may be found in Ref. [1, 7].

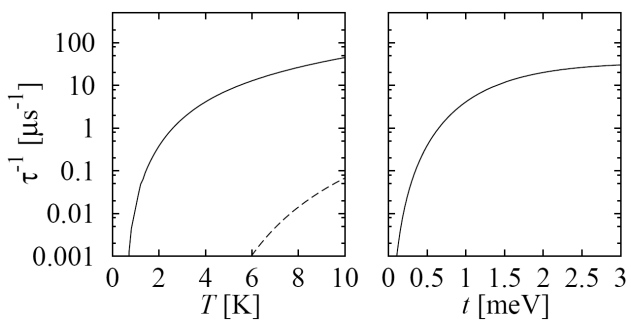

Fig. 1. Dephasing rates as a function of temperature for $t=1 \mathrm{meV}$ (left) and as a function of the tunneling parameter at $T=4 \mathrm{~K}$ (right). Solid lines - pure dephasing due to elastic phonon scattering. Dashed line — dephasing due to real transitions.

The pure dephasing rates corresponding to the two-phonon virtual transition as a function of temperature are shown in the left part of Fig. 1 (solid line). Dephasing rates due to real, single-phonon assisted transitions are shown in the same figure for comparison (dashed line). The tunneling parameter is fixed at $t=1 \mathrm{meV}$. At low temperatures, the single-phonon transition is suppressed due to the fact that phonon energies are much lower than the energy difference between the singlet states. At these temperatures the dominating decoherence mechanism is due to elastic phonon scattering and leads to pure dephasing. This two-phonon process is much less influenced by decreasing temperature since it involves only a virtual transition to the higher energy singlet states. The right part in Fig. 1 shows pure dephasing rates as a function of the tunneling parameter $t$ at $T=4 \mathrm{~K}$. This parameter is responsible for the mixing of the singlet states and for the magnitude of the energy difference between them.

\section{Conclusion}

In conclusion, we have shown that interdot tunneling and the Pauli principle which are necessary for gate oper- ation, lead to electron spin dephasing in DQDs that does not depend on the spin-orbit coupling and is not dependent on the presence of magnetic field. The decoherence processes may result either from single-phonon assisted real transitions between singlet states or from two-phonon assisted virtual transitions. The single-phonon transitions are suppressed at low temperatures. Contrarily, two-phonon assisted transitions cannot be frozen out, and lead to relevant dephasing rates even at low temperatures.

Although carrier-phonon coupling is generally stronger in self-assembled structures, the singlet-triplet dephasing rates are comparable to those for gated dots [7] at the same temperatures. This is due to much larger Coulomb energies which lead to increased energy separation from the doubly occupied states and thus reduce the rate of the second-order process. However, since the self-assembled dots are typically operated at much higher temperatures (a few kelvins, compared to a fraction of a kelvin for gated structures), the relative role of the spin dephasing channel discussed here may be greater in these structures.

\section{Acknowledgments}

This work was supported in part by the Czech Science Foundation (grant no. 202/07/J051).

\section{References}

[1] K. Roszak, A. Grodecka, P. Machnikowski, T. Kuhn, Phys. Rev. B 71, 195333 (2005).

[2] D. Loss, D.P. DiVincenzo, Phys. Rev. A 57, 120 (1998).

[3] D.P. DiVincenzo, D. Bacon, J. Kempe, G. Burkard, K.B. Whaley, Nature 408, 339 (2000).

[4] P. Hawrylak, M. Korkusiński, Solid State Commun. 136, 508 (2005).

[5] R. Hanson, B. Witkamp, L.M.K. Vandersypen, L.H. Willems van Beveren, J.M. Elzerman, L.P. Kouvenhoven, Phys. Rev. Lett. 91, 196802 (2003).

[6] J.M. Elzerman, R. Hanson, L.H. Willems van Beveren, B. Witkamp, L.M.K. Vandersypen, L.P. Kouwenhoven, Nature 430, 431 (2004).

[7] K. Roszak, P. Machnikowski, Phys. Rev. B 80, 195315 (2009).

[8] E.A. Muljarov, R. Zimmermann, Phys. Rev. Lett. 93, 237401 (2004).

[9] E.A. Muljarov, T. Takagahara, R. Zimmermann, Phys. Rev. Lett. 95, 177405 (2005).

[10] K. Hornberger, J.E. Sipe, Phys. Rev. A 68, 012105 (2003).

[11] P. Machnikowski, Phys. Rev. Lett. 96, 140405 (2006).

[12] G.D. Mahan, in: Polarons in Ionic Crystals and Polar Semiconductors, Ed. J.T. Devreese, North-Holland, Amsterdam 1972.

[13] H.-P. Breuer, F. Petruccione, The Theory of Open Quantum Systems, Oxford University Press, Oxford 2002. 